\title{
EFFECTIVE POLARIZATION IN QUASI-FREE SCATTERING
}

\author{
Th.A.J. MARIS, M.R. TEODORO and E.A. VEIT \\ Instituto de Física, Unversidade Federal do Rio Grande do Sul, 90000 Porto Alegre, RS, Brazll
}

Recerved 13 January 1980

Revised manuscript recerved 23 April 1980

A simple relation, involving only experimental quantities, for the effective polarizations in quasi-free ( $p, 2 p$ ) scattering on closed shell nucle1 is tested for recent TRIUMF (p, 2p) experiments with $200 \mathrm{MeV}$ polarized protons.

In quasi-free scattering experiments [1] the ejected nucleon is in general polarized [2] before it is knocked out. There are cases in which the combined influence of the nuclear spin-orbit coupling and the effective absorption by multiple scattering [3] causes this polarization to be nearly complete. It can be measured [4] using a polarized proton beam, because the medium energy $p-p$ cross section is sensitive to relative spin orientations of the bombarding and target protons.

There is a simple prediction which one can make for the case of good shell nuclei like ${ }^{16} \mathrm{O}$ or ${ }^{40} \mathrm{Ca}$, namely, that the average effective polarizations of the protons in two sub-shells, split by the spin-orbit coupling, should vanish $[5,6]$ to a good approximation:

$(l+1) P_{\mathrm{eff}}\left(j=l+\frac{1}{2}\right)+l P_{\mathrm{eff}}\left(j=l-\frac{1}{2}\right)=0$

This relation agrees with actual distorted wave calculations $[4,7]$ but is quite independent of the optical and shell model potentials which generate the distortions and single particle wave functions. In the derivation of relation (1) the impulse and factorization approximations are used and the spin-orbit distortion is neglected, which should in our case introduce only a small error $[7,8]$.

Recently a TRIUMF group $[9,10]$ has performed an extensive series of measurements of the asymmetry

$A=\frac{\mathrm{d} \sigma / \mathrm{d} \Omega_{1} \mathrm{~d} \Omega_{2} \mathrm{~d} E(\uparrow)-\mathrm{d} \sigma / \mathrm{d} \Omega_{1} \mathrm{~d} \Omega_{2} \mathrm{~d} E(\downarrow)}{\mathrm{d} \sigma / \mathrm{d} \Omega_{1} \mathrm{~d} \Omega_{2} \mathrm{~d} E(\uparrow)+\mathrm{d} \sigma / \mathrm{d} \Omega_{1} \mathrm{~d} \Omega_{2} \mathrm{~d} E(\downarrow)}$ for $(p, 2 p)$-scattering on the two $p$-states of ${ }^{16} \mathrm{O}$, for a coplanar geometry with $200 \mathrm{MeV}$ incoming protons with polarizations orthogonal to the scattering plane (normalized to $100 \%$ and denoted by the arrows in definition (2)).

The effective polarization of the nuclear proton involved in the quasi-free reaction can to a good approximation be calculated from the asymmetry, defined in eq. (2), by the relation [6]

$P_{\text {eff }}=(A-P) /\left(C_{\mathrm{nn}}-P A\right)$

Here $P$ and $C_{n n}$ denote the coefficient functions in the expression for the cross section of the scattering of free protons, with polarizations $P_{0}$ and $P_{3}$ orthogonal to the scattering plane $[11,12]$.

$$
(\mathrm{d} \sigma / \mathrm{d} \Omega)_{\text {free }}=I_{0}\left[1+\left(P_{0}+P_{3}\right) P+P_{0} P_{3} C_{\mathrm{nn}}\right]
$$

We have compared the values of $P_{\text {eff }}\left(j=\frac{1}{2}\right)$ and $-2 P_{\text {eff }}\left(j=\frac{3}{2}\right)$, following from eq. (3) and the TRIUMF values [10] for $A$, which should be equal according to relation (1). For the calculation of the functions $P$ and $C_{n n}$ we used the phase shifts of Arndt et al. [13, 14]. We checked several resulting values for $P$ and $C_{\text {nn }}$ directly with available data for free polarized $p-p$ scat. tering. The kinematics of the free process corresponding to the quasi-free one was defined by the initial proton momenta. We have selected kinematical situations for which one expects that the impulse and factorization approximations [1] are good [6] and the off-shell effects small. 
The resulting values for the effective polarizations, in dependence of the angles $\theta_{1}$ of the two outgoing protons with the beam direction and of the kinetic energy $T_{1}$ of one of these protons, are shown in fig. 1. For the cases $\theta_{1}=\theta_{2}$, the agreement is excellent. (Incidentally, in these cases, the experimental asymmetries and calculated effective polarizations should be antisymmetrical in respect to the point $T_{1}=T_{2}$, as follows from the symmetry under a $180^{\circ}$ rotation about the beam direction.) However, there are appreciable differences in the cases $\theta_{1} \neq \theta_{2}$. We have checked that these properties of the curves of fig. 1 are indeed insensitive against off-shell corrections (by comparing the result of initial and final state prescriptions), against the uncertainties in the functions $P$ and $C_{\mathrm{nn}}$ for free scattering and against corrections stemming from the neglected spin-orbit optical potential (by taking the nuclear depolarization into account).

The question then arises, which corrections to the

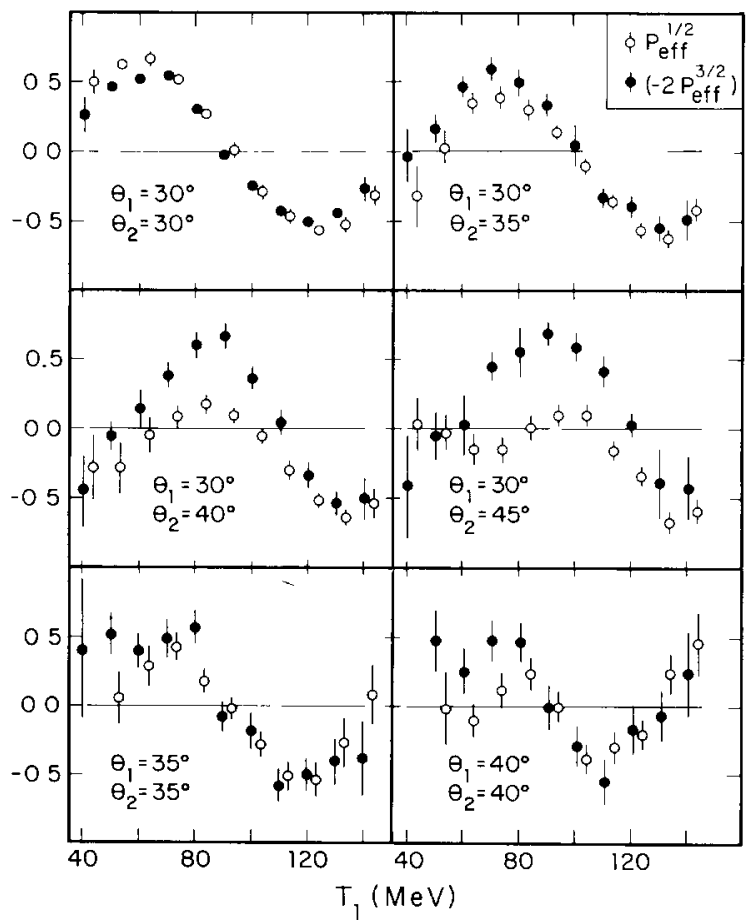

F1g. 1. The effective polarization calculated from TRIUMF measurements [10] of $200 \mathrm{MeV}$ coplanar (p, 2p) scattering on ${ }^{16} \mathrm{O}$, resulting in the $J=\frac{1}{2}$ ground state and the $J=\frac{3}{2}$ first excited state of ${ }^{15} \mathrm{~N}$. The energies and angles with the beam direction of the two outgoing protons are denoted by $T_{1}$ and $\theta_{1}$. factorized DWIA are responsible for the deviations in the $\theta_{1} \neq \theta_{2}$ cases and do not spoil the agreement for $\theta_{1}=\theta_{2}$. Although we have no definite answer to this question, we would like to make a few remarks

(1) We found that a good fit in all cases could be achieved by a strong reduction of the used value of the function $P$, defined in eq. (4). For $\theta_{1}=\theta_{2}$ the value of $P$ is for symmetry reasons anyhow small and therefore the agreement is there maintained. However the assumption that the effective value of $P$ may be strongly reduced for nuclear protons, as compared to free ones, has to be explained and reconciled with the $2 \mathrm{~s}$-knockout in ${ }^{40} \mathrm{Ca}$, where a much smaller reduction is observed [8].

(2) We have not found any other mechanism for the reaction than the quasi-free one, which would explain the observed strongly $j$-dependent asymmetries and it seems to us therefore clear that these are essentially caused by the effective polarizations of the knockedout protons.

(3) The first tematk illustrates the fact that quasifree scattering with polarized protons at suitable geometries may offer the rare possibility to observe quantitatively deviations from the predictions of the factorized DWIA. In nearly all known cases, suspected deviations can be explained away on the basis of uncertainties in the nuclear wave functions and distorting potentials, which may have strong geometry-dependent effects. In our case such uncertainties cancel to a high degree in the ratios involved.

(4) A measurement of the (p,pn) reaction with polarized protons, corresponding to the discussed ( $\mathrm{p}, 2 \mathrm{p}$ )-process, would give a new condition to be fulfilled by any explanation of the observed discrepancies.

We thank Dr.P. Kitching for sending us the TRIUMF data before publication, V.E. Herscovitz and C. Schneider for discussions and the CNPq and FINEP for financial support.

\section{References}

[1] T. Berggren and H. Tyrén, Ann. Rev. Nucl. Sc1. 16 (1966) 153.

[2] Th.A.J. Mar1s, Nucl. Phys. 9 (1958/59) 577.

[3] H.C. Newns, Proc. Phys. Soc. A66 (1953) 477.

[4] Gerhard Jacob, Th.A.J. Maris, C. Schneider and M.R. Teodoro, Phys. Lett. 45B (1973) 181, Nucl. Phys. A257 (1976) 517. 
[5] V.E. Herscovitz, Th.A.J. Marıs and M.R. Teodoro, Phys. Lett. 69B (1977) 33.

[6] Th.A.J. Maris, M.R. Teodoro and C.A.Z. Vasconcellos, Nucl. Phys. A322 (1979) 461.

[7] C. Schneider, Nucl. Phys. A300 (1978) 313.

[8] N.S. Chant, P. Kitchıng, P.G. Roos and L. Antonuk, Phys. Rev. Lett. 43 (1979) 495.

[9] P. Kitchıng et al., Phys. Rev. Lett. 37 (1976) 1600.
[10] P. Kitching, C.A. Miller, W.C. Olsen, D.A. Hutcheon, W.J. McDonald and A.W. Stetz, TRIUMF preprint TRIPP-79-34 (1979).

[11] L. Wolfenstein, Ann. Rev. Nucl. Sc1. 6 (1953) 43.

[12] Clifford R. Schumacher and Hans A. Bethe, Phys. Rev. 121 (1961) 1534.

[13] M.H. McGregor, R.A. Arndt and R.M. Wright, Phys. Rev. 182 (1969) 1714.

[14] R.A. Arndt, R.H. Hackman and L.D. Roper, Phys. Rev. C15 (1977) 1002. 